tion in the larynx. In this condition a nasotracheal tube is passed through highly inflammatory oedematous tissue.

I am convinced that a tracheotomy is by far the safest method of treating these children. The writers have rightly emphasized the importance of the awareness of this condition. May I also emphasize that close co-operation between the paediatrician and laryngologist and anaesthetist is essential in its diagnosis and treatment. May I also emphasize the importance of the work of the coroner's pathologist which has been done largely by Professor Camps in recognizing and drawing the attention of the clinicians to an effective treatment for it.

Lastly, three severe cases of acute epiglottitis have been admitted to the Bradford Children's Hospital over the past eight weeks. It would be interesting to know if other centres in Britain have had a similar experience this winter.-I am, etc.

Ear, Nose, and Throat Unit,

H. MORUS JONES

.

Bradford, Yorks

\section{Vaccination against Rubella}

SIR,-Dr. Constance A. C. Ross (8 January, p. 109) is rightly critical of "widespread attempts" to identify and immunize all seronegative childbearing women. We feel that it is, nevertheless, practicable and desirable to identify and immunize seronegative women (at risk) belonging to certain special groups. In the London borough of Redbridge the schools have been notified that the school health service will willingly provide rubella vaccine to the general practitioners for immunizing the female school teachers at risk. Health visitors, nurses, and midwives are potential carriers-besides being at risk themselves. We are currently engaged in establishing their immune status, and in all cases vaccination is offered, under supervision, to those who are still susceptible.

The emphasis on the present policy of careful serological testing during the early antenatal period of any pregnant woman who is at risk from contact is strongly supported and should, of course, be carried out as soon after contact as possible. We also beg to stress the importance of follow-up and retesting after an interval of four weeks those women whose serum shows absence of or a very low titre of antibodies. A rising titre at this stage is indicative of a subclinical infection. The school girls are offered rubella vaccination at the age of 13 , just as they are in the rest of the country.-We are, etc.,

J. K. ANAND

London Borough of Redbridge

Joan M. POOLEY

Barking Hospital,

W. D. WHITE Barking, Essex

SIR,-Dr. Constance A. C. Ross (8 January, p. 109) raises a point of practical importance when she suggests that the immune status (for rubella) of high-risk non-pregnant workers such as nurses and school teachers should be determined and those seronegative should be immunized. In the West Somerset group of hospitals it has been found that on an average of three or four occasions each year a nurse in the first trimester of pregnancy is in contact with either a baby excreting rubella virus or an adult with a rubella-like rash. It is often a few weeks on these occasions before it can be established whether the nurse's baby is likely to be affected. The pregnant nurse is naturally intensely anxious, especially as she may already have had practical experience of dealing with babies suffering from rubella deformities, or has certainly in her professional training been made aware of the full extent of possible malformations.

To prevent these situations it has been the custom in this group of hospitals for the last two years to assess the rubella complement fixing (C.F.) antibodies of nurses at the initial medical examination. Blood is taken at this time anyway for haemoglobin examination and no additional venepuncture is necessary. Nurses with a C.F. titre $<64$ are offered rubella immunization. This procedure has already averted a number of crises.

As well as testing for antibodies a careful history for rubella is taken and a comparison of history with present susceptibility has been made. Out of a total of 121 tested in the first group 30 were susceptible and 91 immune. Of 52 with a definite history of rubella one was susceptible and 51 were immune (of these, five gave a history of at least two attacks of "rubella"). Of 64 with no history of rubella 29 were susceptible and 35 were immune. Five had an indefinite history of rubella, and all were immune.

These results illustrate the well-known unreliability of a history of rubella. We strongly recommend that the level of rubell antibodies be measured for all female nursing staff when they start training and that those who are seronegative should be offered immunization.-We are, etc.,

Phinip T. Penny Musgrove Park Hospital,

JOHN V. S. PETHER

Taunton

\section{Immune Suppression, Gliomas, and} Tuberculosis

SIR,-There may be a more simple answer to the problem than the theory of immune surveillance, summarized very well by Dr. R. Finn and his team (8 January, p. 111) and the implications may be still more interesting.

In their six cases which had tuberculosis the infection was controlled because the defences against the mycobacteria were fully effective. Provided that the defensive mechanisms are sufficiently prolonged, extrinsic factors such as tuberculosis may possibly be responsible indirectly for many neoplasms. The tumours may be byproducts of the high level of immunity not necessarily evidence of failure of internal surveillance. Unless other primary tumours are excited by the immune irritation the templates of other cells may be unaltered. The intensity of immune response may likewise have made the transplant recipients grow tumours. More instead of less immunosuppression might therefore be advisable. The internal security of their own cases may have been compromised only locally when the gliomas developed.

Sarcoidosis has similarities with tubercu- losis yet its immune pattern is different. Though tuberculosis in Britain is now uncommon sarcoidosis is possibly more frequent. Both diseases sometimes are familial, and occasionally either may precede or follow the other. ${ }^{1}$ In mild cases the diagnosis of both may be missed. It is quite likely therefore that at least another third of the 26 cases with gliomas may have had sarcoidosis instead of tuberculosis. If the cases are still living the lymphocyte test of Caspary and Field would decide this point. ${ }^{2}$

The importance of immunity in sarcoidosis was emphasized five years ago when sarcoid infiltration of endocrine glands was found to be significant. ${ }^{3}$ Function of the glinds can be normal, stimulated, or depressed. Sarcoidosis is reported with coeliac disease, ${ }^{4}$ which carries an increased risk of reticuloses. But other tumours than these (and gliomas) may be associated. In two reported cases, for instance, chronic irritation from hypercalcuria may possibly have caused renal carcinomas. ${ }^{5}$ One of these cases had sarcoidosis and a parathyroid adenoma ${ }^{6}$ and the other in many respects was similar. ${ }^{7}$ Sometimes mammary carcinomas show hypercalcaemia $^{8}$ with, rarely, parathyroid hormonelike activity. ${ }^{9}$ And the finding of noncaseating granulomas at mastectomy may perhaps be more than a coincidence. The active and prolonged cellular immunity of sarcoidosis may sometimes be the common denominator.

Neoplasms are also associated with immune deficiehcy, and the atrophic process extends beyond only the $B$ and $T$ cells. $A$ recent example, ${ }^{10}$ improved by two thymic transplants, still had Addison's disease, pernicious anaemia, and the coeliac syndrome. The "autoantibodies" which had earlier been inappropriately active probably caused the secretory failure of the adrenals, jejunum, stomach, and possibly the thymus. An exogenous agent possibly started the immune reactions, and the initial inflammation may have been sarcoidosis. Glandular failure in the face of intact defences may be peculiar to sarcoidosis.-I am, etc.,

S. Luke's Hospital,

G. A. MACGREgor Guildford,

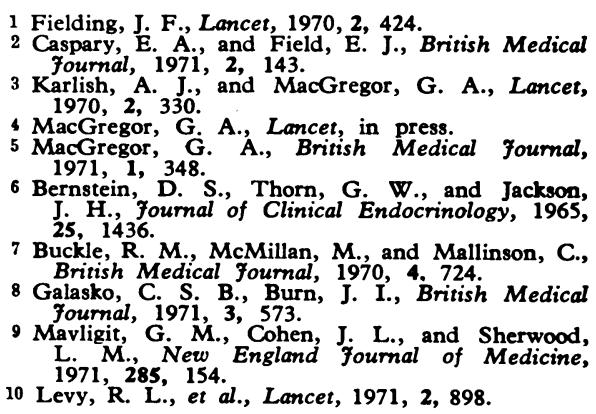

Sir,-Referring to the letter by Dr. R. Finn and others (8 January, p. 111), my own interest lies more in the association of tuberculosis and demyelinating disease. In our practice between 1936 and 1940 five cases of florid and fatal pulmonary tuberculosis were nursed at home by apparently healthy adults, all of whom subsequently developed disseminated sclerosis and have died of intercurrent infection. Three of these 